\title{
BOOKS
}

\section{Practical approach to issues in virology} Medical Virology: A Practical Approach, edited by U Desselberger (1995). Oxford University Press, 70 Wynford Drive, Don Mills, Ontario M3C 1J9. ISBN 0-19-963330-4; 214 pages; $\$ 98.95$

This textbook is part of a series of books addressing a wide variety of biological and molecular issues in science. Medical Virology: A Practical Approach consists of eight chapters on practical aspects of virology. As the title indicates, the text provides protocols and suggestions regarding laboratory virology and the clinical diagnosis of virological infections. The text is not intended to serve as a comprehensive reference, but rather outlines topical issues in virology. Chapter 1 consists of a quick review of traditional techniques of viral diagnosis. For persons active in the field of laboratory virology this chapter illustrates how much the field of clinical virology has changed in the past 10 years. At the end of the chapter, the author, CR Madeley, states that "tradition should not be discarded lightly". However, as the detection of viruses migrates to shell vial centrifugation cultures and nucleic acid amplification, one wonders how much of traditional virology will remain given the need and the ability to provide more rapid, standardized and cost effective virological testing with the newer molecular techniques.

The second chapter provides an overview of current immunoassay technologies by addressing different assay designs and procedures. These procedures are both practical and useful, especially if one wants to produce in-house assays to specific targets. Relatively little attention is focused on the impact of large scale automation and random access analyzers that will result in 'economies of scale' when coupled to efficient transport and computerized reporting. Modern technology will revolutionize the virology laboratory of the future and will likely favour the existence of larger laboratories over small scale hospitalbased testing due to potentially lower costs per test.

Chapters 3 and 5 review nucleic acid detection by hybridization and the polymerase chain reaction (PCR). Numerous protocols and suggestions are included and the illustrations used are applied to clinical virology. Although the strengths and weaknesses of each of the technologies are well described and multiple methods of cell, serum, plasma and tissue extraction are provided, a greater emphasis on the importance of standardization of methodology could have been included. As the application of this type of technology becomes more and more widespread, it is critical for laboratory experts to recognize that, with careful attention to detail, in-house methods can be standardized to yield reproducible assays. However, without standardized reagents for extraction, amplification, detection and well characterized standards, interlaboratory results are poorly reproducible and accurate clinical correlations between centres are difficult. The introduction of commercial PCR assays has introduced new levels of standardization with dramatic improvements in centre to centre reproducibility for both qualitative and quantitative PCR results.

Chapter 4 discusses the application of immunoblot assays in virology providing both protocols and practical hints for success. Recombinant and synthetic peptides combined with immunoblot assays have revolutionized the manufacture of reliable and sensitive immunoassays because they speed optimization and allow organism- and antigen-specific confirmation of serological results. The design and testing of antiviral compounds is addressed in chapter 6 , which provides insights into computer-based drug design and the assays used to test drug effectiveness. Human immunodeficiency virus is used as a model virus, and a variety of techniques to assess antiviral efficacy are described. The focus of the chapter is on in vitro assays for virus susceptibility, and issues pertaining to viral load testing from patients receiving antiviral treatment are not addressed.

The last two chapters deal with the new and emerging field of molecular epidemiology and sequence-based evolutionary analysis of viruses. These areas complement each other and will be particularly important as the technology for viral- and gene-based sequencing becomes more universal. In particular, nucleic acid sequencing permits one to trace the evolutionary similarity between viruses and improves the accuracy of taxonomy. This sequence information can also be used to detect new viral agents by targeting conserved sequences between different viral groups. In addition, PCR-based automated sequencing of hypervariable regions has already revolutionized molecular epidemiology of outbreaks and can address the role of viral quasispecies during normal viral replication. Included in the chapter on evolutionary analysis of viruses are important discussions on the strengths and weaknesses of the different types of analytical techniques as well as information on how to obtain software via the internet.

In summary, this text is indeed practical and provides helpful hints for the practising virologist, pathologist, student or molecular biologist. This is not a comprehensive text, but unlike most comprehensive texts it is one that will be read.

Mel Krajden MD FRCPC Virology and Serology, The Toronto Hospital Toronto, Ontario 


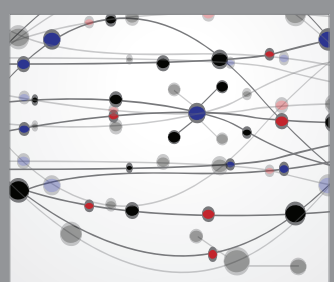

The Scientific World Journal
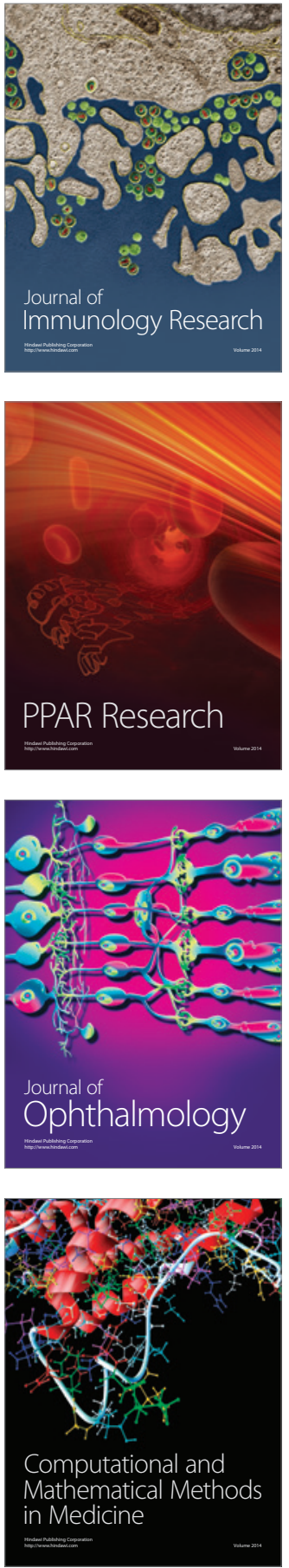

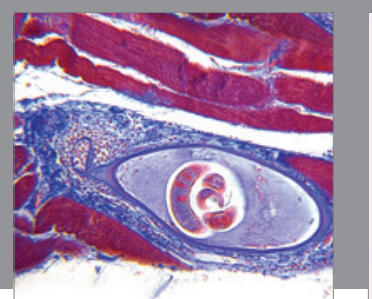

Gastroenterology Research and Practice

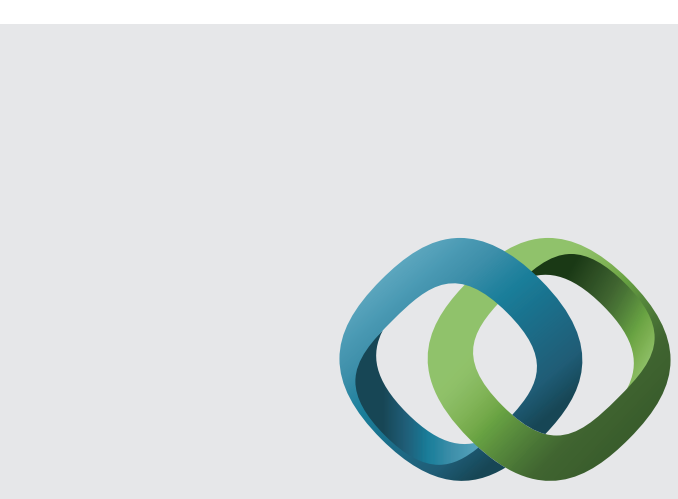

\section{Hindawi}

Submit your manuscripts at

http://www.hindawi.com
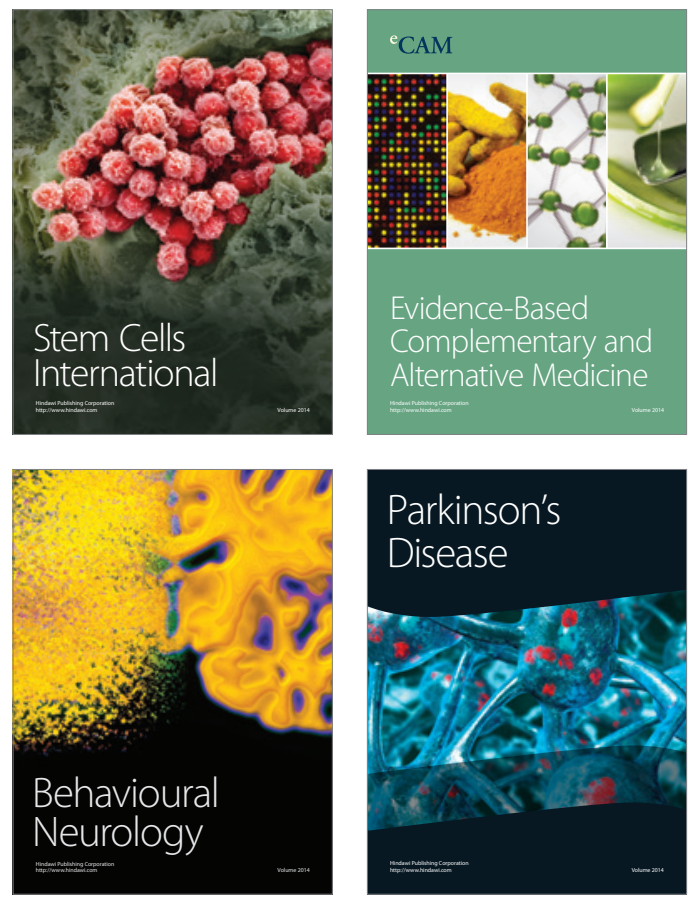
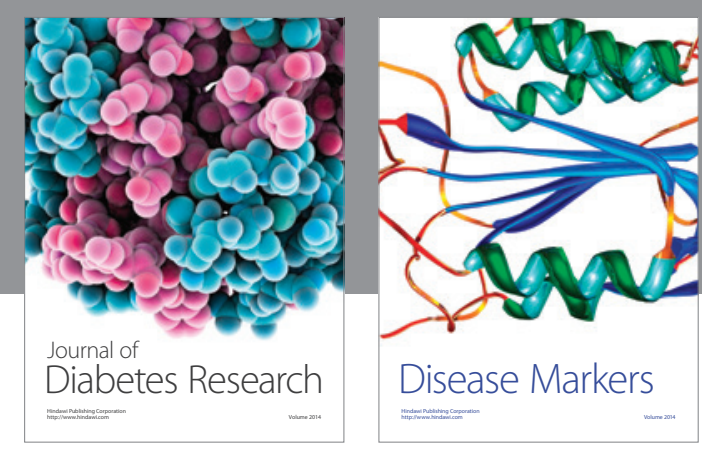

Disease Markers
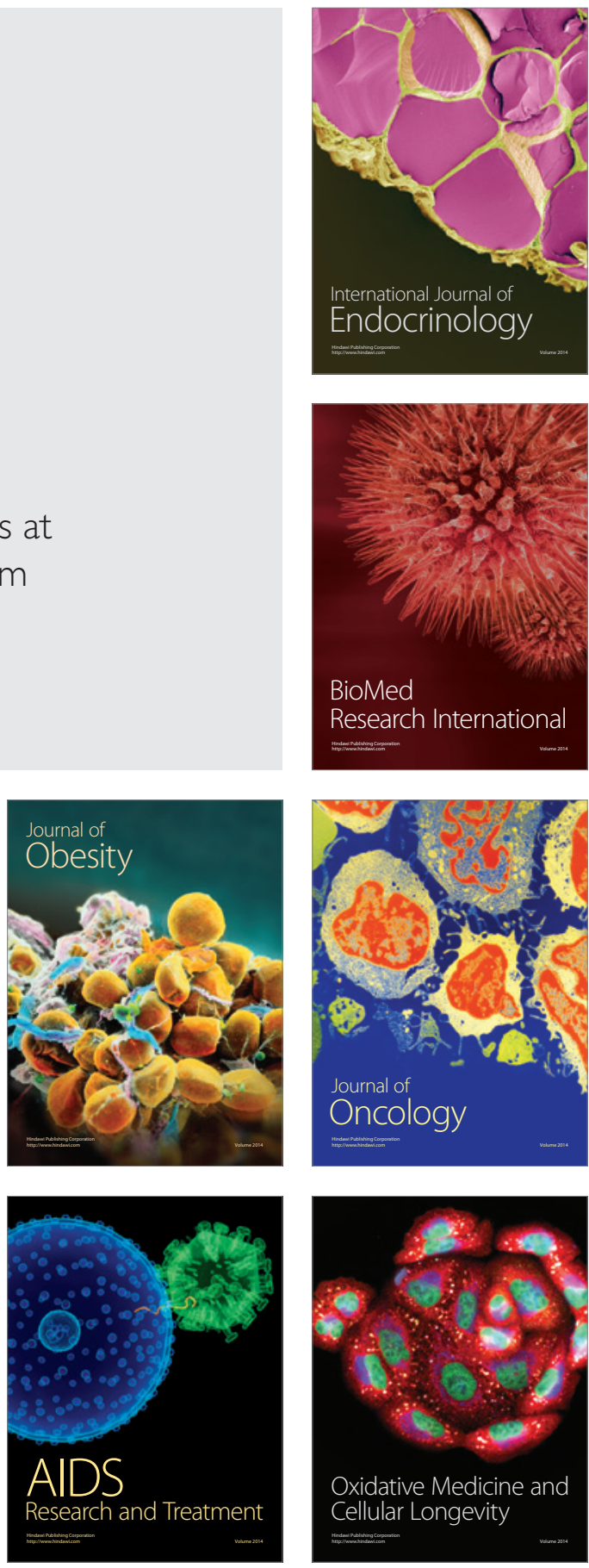